PDES, SUBMANIFOLDS AND

AFFINE DIFFERENTIAL GEOMETRY

BANACH CENTER PUBLICATIONS, VOLUME 69

INSTITUTE OF MATHEMATICS

POLISH ACADEMY OF SCIENCES

WARSZAWA 2005

\title{
SOME RESULTS ON PROJECTIVELY FLAT AFFINE SURFACES
}

\author{
ANTONIO MARTÍNEZ and FRANCISCO MILÁN \\ Departamento de Geometría y Topología \\ Facultad de Ciencias, Universidad de Granada \\ 18071 Granada, Spain \\ E-mail: amartine@ugr.es,milan@ugr.es
}

\begin{abstract}
We focus our attention on projectively flat affine surfaces. First, we classify the affine surfaces with projectively flat induced connection and constant Pick invariant. We also investigate the compact case and study how the geometry at the boundary determines the geometry of the surface.
\end{abstract}

1. Introduction. The properties of nondegenerate surfaces invariant under the group of unimodular affine transformations in the real affine three-space $\mathbb{R}^{3}$ were first treated by Tzitzèica, [18], and later by Blaschke, [2]. On such surfaces there is a canonical transversal vector field (Blaschke normal) which induces an affine connection, a bilinear symmetric form (Blaschke metric) and a volume form.

In this paper we investigate those surfaces for which the induced connection is projectively flat; they are called projectively flat affine surfaces. Previous papers on this subject are [9], [13], [14] and [15] among others, [1] appeared recently. Basic examples of projectively flat affine surfaces are the affine spheres and all locally symmetric surfaces. A nondegenerate immersion of a surface in $\mathbb{R}^{3}$ is called an affine sphere if all the Blaschke normal lines through each point of the surface either are parallel or else intersect at one point. There exist, surprisingly, many examples of affine spheres which have been widely studied since the beginning of the past century. Some interesting results can be found for example in [2], [8], [10], [11] [12] and [19]. However there are examples of projectively flat surfaces which are not locally symmetric neither affine spheres (see [15]). Although one is far from a local classification there are known local classifications under further restrictions like flat Blaschke metric, see ([9]).

2000 Mathematics Subject Classification: Primary 53A15; Secondary 53B20, 53B30.

Research partially supported by MCYT-FEDER Grant No. BFM2001-3318 and Junta de Andalucía CEC: FQM0203.

The paper is in final form and no version of it will be published elsewhere. 
In the second section we describe the fundamental invariants in affine differential geometry and give some basic examples of projectively flat affine surfaces.

In Section 3, we completely classify projectively flat nondegenerate immersions with constant Pick invariant (see Theorem 1 and Theorem 2).

In Section 4, we investigate compact locally strongly convex surfaces with boundary and projectively flat induced connection. We study the question: how does the geometry at the boundary determine the geometry of the surface?

We learned from the referee's report that the results in Section 3 have been proved in [1] using a different approach.

2. Basic notations and examples. By $\Sigma$ we always mean a connected surface with boundary $\partial \Sigma$. For a nondegenerate immersion $\psi: \Sigma \rightarrow \mathbb{R}^{3}$ in the affine space $\mathbb{R}^{3}$ equipped with its usual flat connection $D$ and volume form given by the determinant function, it is well known, (see [12]), how to introduce a canonical transversal vector field $\xi$ (Blaschke normal) and how, using $\xi$, by the formulas of Gauss and Weingarten we obtain the affine metric, $g$, the induced connection, $\nabla$, and the shape operator, $S$.

However, some of the equiaffine invariants can also be introduced using "Euclidean" invariants. Indeed by considering on $\mathbb{R}^{3}$ its canonical Euclidean structure, the affine metric, also known as Berwald-Blaschke metric, is the following semi-Riemannian metric $g$ on $\Sigma$,

$$
g=\left|K_{e}\right|^{-1 / 4} \sigma_{e}
$$

where $\sigma_{e}$ and $K_{e}$ are the second fundamental form and the euclidean Gauss curvature of $\psi$, respectively. The equiaffine normalization of $\psi$ is given by the Blaschke normal $\xi$ and the affine conormal vector field $N$ :

$$
\xi=\frac{1}{2} \triangle_{g} \psi, \quad N=\left|K_{e}\right|^{-1 / 4} n_{e},
$$

where $\triangle_{g}$ is the Laplace-Beltrami operator associated to $g$ and $n_{e}$ denotes the usual Gauss map of $\psi$. With this normalization,

$$
g:=-\langle d \psi, d N\rangle
$$

here, $\langle.,$.$\rangle is the usual scalar product in \mathbb{R}^{3}$.

The structure equations of the immersion read

$$
\begin{array}{ll}
\text { Gauss : } & D_{X} d \psi(Y)=d \psi\left(\nabla_{X} Y\right)+g(X, Y) \xi, \\
& D_{X} d N(Y)=d N\left(\bar{\nabla}_{X} Y\right)+\sigma(X, Y) N ; \\
\text { Weingarten: } & d \xi(X)=-d \psi(S(X)),
\end{array}
$$

where $X, Y, \ldots$ here and in the following are tangent vector fields on $\Sigma$. It is well known that the Levi-Civita connection of $g$ is $(\nabla+\bar{\nabla}) / 2$. The difference tensor $2 K:=\nabla-\bar{\nabla}$ defines the totally symmetric cubic form $\hat{K}(X, Y, Z):=g(K(X, Y), Z) ; K$ satisfies the apolarity condition trace $K_{X}=0$ and defines the Pick invariant $2 J:=\|K\|^{2}$, where the norm is induced by $g$ on tensor spaces. The affine shape operator $S$ gives the symmetric Weingarten form $\sigma(X, Y):=g(S X, Y)$.

The basic surfaces in this theory are the affine spheres. A nondegenerate immersion $\psi$ is called an affine sphere if all affine normal lines of the immersion either intersect at one 
point, called its center, or else are parallel. It is called elliptic, parabolic or hyperbolic according to whether the center is, respectively, on the concave side of the surface $\psi(\Sigma)$, at infinity, or on the convex side. The prototypes of affine spheres of the three types are given by quadrics: ellipsoids, paraboloids and hyperboloids, which are characterized as nondegenerate immersions with $K=0$ (Theorem of Maschke).

The surface $\psi_{1} \psi_{2} \psi_{3}=1$ is, besides the elliptic paraboloid, the unique affine sphere with flat definite Blaschke metric (see [8]). In the indefinite case, the surfaces $\psi_{3}=$ $\psi_{1} \psi_{2}+\Phi\left(\psi_{2}\right)$, where the differentiable function $\Phi$ depends only on the second coordinate function $\psi_{2}$, and $\psi_{1}\left(\psi_{2}^{2}+\psi_{3}^{2}\right)=1$ are the unique affine spheres with flat indefinite Blashcke metric (see [11]).

The immersion $\psi$ is projectively flat if the induced connection $\nabla$ is projectively equivalent to a flat connection. This condition is equivalent to either trace ${ }_{g} \nabla=0$ or $\triangle_{g} \xi \| \xi$ (see $[12])$.

From the fundamental Theorem given in [5], every projectively flat immersion has a dual immersion (also projectively flat) $\bar{\psi}$ with Blaschke metric metric $g$ and induced connection $\bar{\nabla}$. Thus, $\bar{K}=\bar{\nabla}-\nabla=-K$ and $\psi$ and $\bar{\psi}$ have the same Pick invariant, the same affine Gauss curvature and the same affine mean curvature (see (5) below and [12] for relations between them)

\section{Projectively flat immersions with constant Pick invariant}

3.1. The definite case. Let $\psi: \Sigma \rightarrow \mathbb{R}^{3}$ be a locally strongly convex immersion, oriented so that the second fundamental form is positive definite everywhere. On $\Sigma$ we have an orientation and a conformal structure representable by the common conformal equivalence class of $g$. Therefore, $\Sigma$ naturally can be regarded as a Riemann surface. Moreover, the structure equations of affine differential geometry can be derived in terms of a local complex parameter $z$ in the following way: if

$$
g:=2 \rho|d z|^{2},
$$

we can introduce complex valued functions $U$ and $B$ and a real function $H$ such that

$$
\begin{array}{ll}
\text { Gauss: } & \psi_{z z}=(\log \rho)_{z} \psi_{z}+U \psi_{\bar{z}}, \\
& \psi_{z \bar{z}}=\rho \xi, \\
\text { Weingarten: } & \xi_{z}=-H \psi_{z}+B \psi_{\bar{z}},
\end{array}
$$

where, for instance, $\psi_{z}$ denotes the partial derivative of $\psi$ with respect to $z$ and ${ }^{-}$is the usual conjugation (see [3], [10] and [20] for more details).

The function $H:=(\operatorname{trace} S) / 2$ is the affine mean curvature and $J=\rho^{-1} U \bar{U}$ is the Pick invariant. The Weingarten form, can be expressed by

$$
\sigma:=-\rho B(d z)^{2}+2 H d z d \bar{z}-\rho \bar{B}(d \bar{z})^{2} .
$$

Using that $\psi_{z z \bar{z}}=\psi_{z \bar{z} z}$ and $\xi_{z \bar{z}}$ is real, from (5) we have the following integrability equations:

$$
\begin{array}{ll}
\text { Gauss: } & (\log \rho)_{z \bar{z}}+U \bar{U}+\rho H=0 ; \\
\text { Codazzi: } \quad & U_{\bar{z}}+U(\log \rho)_{\bar{z}}=\rho B, \\
& \rho U \bar{B}-H_{z} \rho=(B \rho)_{\bar{z}} .
\end{array}
$$


From (5) and (9) we also get

$$
\xi_{z \bar{z}}=\rho^{-1}(\bar{B} \rho)_{z} \psi_{z}+\rho^{-1}(B \rho)_{\bar{z}} \psi_{\bar{z}}-H \rho \xi,
$$

and consequently, the immersion is projectively flat if and only if $B \rho$ is holomorphic, that is, the $(2,0)$-part of the Weingarten form $\sigma$ must be holomorphic.

THEOREM 1. Let $\psi: \Sigma \rightarrow \mathbb{R}^{3}$ be a locally strongly convex immersion with projectively flat induced connection and constant Pick invariant. Then $\psi$ is locally equiaffine-equivalent to a quadric or to the surface $\psi_{1} \psi_{2} \psi_{3}=1$.

Proof. If $J=0$, then from the Maschke Theorem, $\psi(\Sigma)$ lies on a quadric.

Assume $J=\rho^{-1}\|U\|^{2}$ is a non-zero constant. Since $B \rho$ is holomorphic, then from (8) and (9) we have

$$
J\left(\log \left(U\|U\|^{2}\right)\right)_{\bar{z}}=H_{\bar{z}}
$$

and hence $\left(\log \left(U\|U\|^{2}\right)\right)_{z \bar{z}}=\left(\log \left(\bar{U}\|U\|^{2}\right)\right)_{z \bar{z}}$, that is, $(\log (U / \bar{U}))_{z \bar{z}}=0$, and we can write

$$
U=e^{\mu+i \nu}
$$

for some real functions $\mu$ and $\nu$, with $\nu$ harmonic. From (8) and (12), $U(3 \mu+i \nu)_{\bar{z}}$ is holomorphic, that is,

$$
(\mu+i \nu)_{\bar{z}}(3 \mu+i \nu)_{\bar{z}}+(3 \mu+i \nu)_{\overline{z z}}=0 .
$$

Using (7) and (11), we have $-H=2 J e^{-2 \mu} \mu_{z \bar{z}}+J$ and

$$
\left(2 e^{-2 \mu} \mu_{z \bar{z}}+3 \mu+i \nu\right)_{\bar{z}}=0 .
$$

Thus,

$$
\mu_{z \bar{z} \bar{z}}=2 \mu_{\bar{z}} \mu_{z \bar{z}}-\frac{1}{2} e^{2 \mu}(3 \mu+i \nu)_{\bar{z}}
$$

Deriving (13) with respect to $z$ and using (15), we get

$$
-\mu_{z \bar{z}}(6 \mu+i 4 \nu)_{\bar{z}}=6 \mu_{\bar{z}} \mu_{z \bar{z}}-\frac{3}{2} e^{2 \mu}(3 \mu+i \nu)_{\bar{z}}
$$

Thus, (14) and the last equation give

$$
0=(3 \mu+i \nu)_{\bar{z}}\left(\frac{8}{3} e^{-2 \mu} \mu_{z \bar{z}}-1\right)=(3 \mu+i \nu)_{\bar{z}}\left(h-\frac{4}{3}(3 \mu+i \nu)\right),
$$

for some holomorphic function $h$. Hence, $2(3 \mu+i \nu)^{2}-3 h(3 \mu+i \nu)$ must be holomorphic and so $3 \mu+i \nu$.

Therefore, $\mu$ is harmonic, $g$ is flat, and we can choose a local parameter $z$ so that $\rho=J^{-1} e^{2 \mu}=1$ and $\nu$ must be constant. We conclude from (8) and (12) that $B=U_{\bar{z}}=0$ and $\psi$ is a flat affine sphere with a non-zero constant Pick invariant. The result follows from $[8]$.

3.2. The indefinite case. Let $\psi: \Sigma \rightarrow \mathbb{R}^{3}$ be a nondegenerate immersion with indefinite Blaschke metric and consider on $\Sigma$ the natural structure of a Lorentz surface induced by $g$. Choose local asymptotic parameters $u$ and $v$ on $\Sigma$ such that

$$
g:=2 \rho d u d v \text {. }
$$


We can introduce real functions $U, V, A, B$ and $H$ satisfying

$$
\begin{array}{ll}
\text { Gauss: } & \psi_{u u}=(\log \rho)_{u} \psi_{u}+U \psi_{v}, \\
& \psi_{v v}=V \psi_{u}+(\log \rho)_{v} \psi_{v}, \\
& \psi_{u v}=\rho \xi ; \\
\text { Weingarten: } & \xi_{u}=-H \psi_{u}+B \psi_{v}, \\
& \xi_{v}=A \psi_{u}-H \psi_{v},
\end{array}
$$

where, for instance, $\psi_{u}$ denotes the partial derivative of $\psi$ with respect to $u$ (see [2] and [19] for more details).

The function $H$ is the affine mean curvature and $J=\rho^{-1} U V$ is the Pick invariant. The Weingarten form can be expressed by

$$
\sigma:=-\rho B(d u)^{2}+2 H d u d v-\rho A(d v)^{2} .
$$

Using $\psi_{u u v}=\psi_{u v u}, \psi_{v v u}=\psi_{u v v}$, and $\xi_{u v}=\xi_{v u}$, from (17) we have the following integrability equations:

$$
\begin{array}{ll}
\text { Gauss: } & (\log \rho)_{u v}+U V+\rho H=0 ; \\
\text { Codazzi: } & U_{v}+U(\log \rho)_{v}=\rho B, \\
& V_{u}+V(\log \rho)_{u}=\rho A, \\
& \rho B V-H_{v} \rho=(A \rho)_{u}, \\
& \rho A U-H_{u} \rho=(B \rho)_{v} .
\end{array}
$$

From (17) and (19) we also have

$$
\xi_{u v}=\rho^{-1}(A \rho)_{u} \psi_{u}+\rho^{-1}(B \rho)_{v} \psi_{v}-H \rho \xi,
$$

and consequently, the immersion is projectively flat if and only if

$$
(B \rho)_{v}=(A \rho)_{u}=0 .
$$

From the above relations and similarly to Theorem 1 we can prove

TheOREM 2. Let $\psi: \Sigma \rightarrow \mathbb{R}^{3}$ be a nondegenerate immersion with indefinite Blaschke metric, projectively flat induced connection and constant Pick invariant. Then $\psi$ is locally equiaffine-equivalent to one of the following surfaces:

i) The ruled surface given by $\psi(u, v)=u \phi(v)+\varphi(v)$, where $\phi$ and $\varphi$ are curves in $\mathbb{R}^{3}$ satisfying $\operatorname{det}\left[\phi, \varphi^{\prime}, \phi^{\prime}\right]=1$ and $\operatorname{det}\left[\phi, \phi^{\prime}, \phi^{\prime \prime}\right]=$ constant .

ii) The affine sphere $\psi_{1}\left(\psi_{2}^{2}+\psi_{3}^{2}\right)=1$.

iii) The affine sphere $\psi_{3}=\psi_{1} \psi_{2}+\Phi\left(\psi_{2}\right)$, where the differentiable function $\Phi$ depends only on the second coordinate function $\psi_{2}$.

4. Compact projectively flat immersions. Along this section $\Sigma$ will be a compact surface and $\psi: \Sigma \rightarrow \mathbb{R}^{3}$ a locally strongly convex immersion with projectively flat induced connection.

If $\partial \Sigma=\emptyset$, then $\Sigma$ is diffeomorphic to the 2-sphere and the holomorphic function $B \rho$ vanishes identically on $\Sigma$ (see [7]). Hence and from (5), $S=H I$, that is, $\psi$ is an affine sphere. Now, (see [2]), $\psi(\Sigma)$ must be an ellipsoid. Consequently, we shall assume in the rest of this section that $\partial \Sigma$ is a non-empty set such that $\psi(\partial \Sigma)=\Gamma$. 
LEMma 1. If $\Gamma$ lies on a plane $\Pi$ and $\Pi \cap \psi(\Sigma \backslash \partial \Sigma)=\emptyset$, then $\psi$ is an embedding and $\psi(\Sigma)$ lies on the boundary of a convex set in $\mathbb{R}^{3}$.

Proof. Assume that $\Gamma$ lies on the $x y$-plane and consider the projective transformation $T$ given by

$$
(x, y, z) \rightarrow T(x, y, z)=(x / z, y / z, 1 / z) .
$$

Then $T \circ \psi$ maps $\Sigma \backslash(\partial \Sigma)$ to a complete surface without boundary. Since projective transformations preserve the sign of curvature, $T \circ \psi$ is a complete locally strongly convex immersion without boundary. Using van Heijenjoort's theorem (see [6]), $(T \circ \psi)(\Sigma \backslash(\partial \Sigma))$ is the boundary of a convex set in $\mathbb{R}^{3}$. Further, $T$ preserves line segments, and therefore sends convex bodies to convex bodies which concludes the proof.

Proposition 1. If the Euler-Poincaré characteristic of $\Sigma, \chi(\Sigma)$, is 1 and $\Gamma$ is an affine line of curvature (not necessarily planar), then $\psi$ is an affine sphere.

Proof. Since $\chi(\Sigma)=1$ the surface $\Sigma$ is conformally equivalent to the closed unit disk $D=\{z \in \mathbb{C} /|z| \leq 1\}$.

Thus, we can consider $\Sigma=D$ and choose polar coordinates $(r, \theta)$ given by $z=u+i v=$ $r e^{i \theta}$. Therefore,

$$
\begin{gathered}
\frac{\partial}{\partial r}=\cos \theta \frac{\partial}{\partial u}+\sin \theta \frac{\partial}{\partial v}, \\
\frac{\partial}{\partial \theta}=-\sin \theta \frac{\partial}{\partial u}+\cos \theta \frac{\partial}{\partial v},
\end{gathered}
$$

on $\partial D$. Using that $(u, v)$ are conformal parameters we have

$$
g\left(\frac{\partial \psi}{\partial r}, \frac{\partial \psi}{\partial \theta}\right)=0
$$

From the above equation and the fact that $\Gamma$ is a line of curvature, we obtain

$$
0=\sigma\left(\frac{\partial \psi}{\partial r}, \frac{\partial \psi}{\partial \theta}\right)=-\frac{1}{2} \sin 2 \theta(e-g)+\cos 2 \theta f \quad \text { on } \partial D
$$

where

$$
e=\sigma\left(\frac{\partial \psi}{\partial u}, \frac{\partial \psi}{\partial u}\right), \quad f=\sigma\left(\frac{\partial \psi}{\partial u}, \frac{\partial \psi}{\partial v}\right) \quad \text { and } \quad g=\sigma\left(\frac{\partial \psi}{\partial v}, \frac{\partial \psi}{\partial v}\right) .
$$

Since the (2,0)-part of $\sigma$ is holomorphic, $F(z)=e(z)-g(z)-2 i f(z)$ is a holomorphic function and so it is $G(z)=z^{2} F(z)$. But the imaginary part of $G$ is a harmonic function on $D$ and it is given by $\sin 2 \theta(e-g)-2 \cos 2 \theta f$ on $\partial D$. Then, from (22) the imaginary part of $G$ vanishes and $G$ must be constant.

As $G(0)=0$, then $F=0$, that is, $B \rho=0$ and $\psi$ is an affine sphere.

Corollary 1. If $\Gamma$ lies on a plane $\Pi$ such that $\Pi \cap \psi(\Sigma \backslash \partial \Sigma)=\emptyset$ and $\Gamma$ is an affine line of curvature, then $\psi$ must be an affine sphere.

Proof. From Lemma 1, the Euler-Poincaré characteristic of $\Sigma$ must be 1 and the Corollary follows from Proposition 1.

Corollary 2. If $\Gamma$ lies on a plane $\Pi$ such that $\Pi \cap \psi(\Sigma \backslash \partial \Sigma)=\emptyset$ and the affine normal lines on $\partial \Sigma$ intersect at one point or are parallel, then $\psi$ is an affine sphere. 
Proof. If the affine normal lines on $\partial \Sigma$ intersect at one point or are parallel, then $\Gamma$ is an affine line of curvature and the result follows from Corollary 1.

\section{References}

[1] T. Binder, Projectively flat affine surfaces, J. Geom. 79 (2004), 31-45.

[2] W. Blaschke, Vorlesungen über Differentialgeometrie II: Affine Differentialgeometrie, J. Springer, Berlin, 1923.

[3] E. Calabi, Convex affine maximal surfaces, Results Math. 13 (1988), 209-223.

[4] F. Dillen, A. Martínez, F. Milán, F. G. Santos and L. Vrancken, On the Pick invariant, the affine mean curvature and the Gauss curvature of affine surfaces, Results Math. 20 (1991), 622-642.

[5] F. Dillen, K. Nomizu and L. Vrancken, Conjugate connections and Radon's Theorem in affine differential geometry, Mh. Math. 109 (1990), 221-235.

[6] J. van Heijenoort, On locally convex manifolds, Comm. Pure Appl. Math. 5 (1952), 223242.

[7] H. Hopf, Differential Geometry in the Large, Lecture Notes in Math. 1000 (1983).

[8] A. M. Li and G. Penn, Uniqueness theorems in affine differential geometry II, Results in Math. 13 (1988), 308-317.

[9] C. Lee and L. Vrancken, Projectively flat affine surfaces with flat affine metric, J. Geom. 70 (2001), 85-100.

[10] A. M. Li, U. Simon and G. Zhao, Global Affine Differential Geometry of Hypersurfaces, Walter De Gruyter, Berlin-New York, 1993.

[11] M. Magid and P. Ryan, Flat affine spheres in $\mathbb{R}^{3}$, Geom. Dedicata 33 (1990), 277-288.

[12] K. Nomizu and T. Sasaki, Affine Differential Geometry, Cambridge University Press, 1994.

[13] B. Opozda, A class of projectively flat surfaces, Math. Z. 219 (1995), 77-92.

[14] B. Opozda, On the realizability of projectively flat connections on surfaces, J. Geom. 70 (2001), 133-138.

[15] F. Podesta, Projectively flat surfaces in $\mathbb{A}^{3}$, Proc. Amer. Math. Soc. 119 (1993), 255-260.

[16] J. Radon, Zur Affingeometrie der Regelflächen, Leipziger Berichte 70 (1918), 147-155.

[17] U. Simon, Local classification of twodimensional affine spheres with constant curvature metric, Diff. Geom. and Appl. 1 (1991), 123-132.

[18] G. Tzitzèica, Sur une nouvelle classe de surfaces, Rend. Circ. Mat. Palermo 25 (1908), 180-187.

[19] P. A. Schirokow and A. P. Schirokow, Affine Differentialgeometrie, Teubner, Leipzig, 1962.

[20] C. P. Wang, Some examples of complete hyperbolic affine 2-spheres in $\mathbb{R}^{3}$, in: Proc. Conf. Global Diff. Geometry Global Analysis (Berlin, 1990), Lecture Notes Math. 1481, Springer, 1991, 272-280. 